\title{
Comparison of minimally invasive versus conventional open harvesting technique for iliac bone graft in secondary alveolar bone grafting in cleft palate patients: a systematic review
}

\author{
Aditi Saha, Sonal Shah, Pushkar Waknis, Prathamesh Bhujbal, Sharvika Aher, Vibha Vaswani \\ Department of Oral and Maxillofacial Surgery, Dr. D. Y. Patil Vidyapeeth, Pimpri, Pune, India
}

\begin{abstract}
J Korean Assoc Oral Maxillofac Surg 2019;45:241-253)
This study evaluated and compared the donor site morbidity following minimally invasive and conventional open harvesting of iliac bone for secondary alveolar bone grafting in cleft palate patients. A thorough electronic search of PubMed, Google Scholar, EMBASE, and an institutional library and manual search of various journals was done; Inclusion criteria: 1) full-text articles using a minimally invasive or conventional open harvesting technique for iliac bone for secondary alveolar grafting in cleft palate patients and 2) articles published between January 1, 2001 and June 30, 2017 and Exclusion criteria: 1) articles published in languages other than English, 2) case reports, case series, animal studies, in vitro studies, and letters to the editor, and 3) full-text article unavailable even after writing to the authors. Preliminary screening of 274 studies excluded 223 studies for not meeting the eligibility criteria. Of the remaining 51 studies, 19 were removed for being duplicates. Of the remaining 32 studies, 15 were excluded after reading the abstract. Of the 17 studies that were left, 2 were excluded because they were in a language other than English, and 2 were excluded because the study group did not mention cleft palate patients. Thus, 13 studies providing results for a total of 654 patients were included in this qualitative synthesis. Minimally invasive bone graft harvest techniques are better than the conventional open iliac bone harvest method because they offer shorter operative time, decreased requirement for pain medications, less pain on discharge, and a shorter hospital stay.
\end{abstract}

Key words: Iliac crest, Cleft palate, Grafting, Bone transplantation

[paper submitted 2018. 10. 26 / revised 2019. 1. 5 / accepted 2019. 1. 9]

\section{Introduction}

With the emergence of new reconstruction techniques, the demand for bone grafting, for repair or as a replacement material or extension of the native anatomy, has increased during the past decade. The quality and quantity of bone graft material needed is largely dictated by the recipient site. Secondary alveolar cleft bone grafting is an integral part of contemporary rehabilitation for patients with cleft lip and palate, and any cleft patient with an alveolar defect should be considered for bone grafting ${ }^{1}$. Restoring the continuity of the maxillary

\footnotetext{
Sonal Shah

Department of Oral and Maxillofacial Surgery, Dr. D. Y. Patil Vidyapeeth, Sant Tukaram Nagar, Pimpri, Pune 411018, India

TEL: +91-9833459822 FAX: +91-20-27423427

E-mail: sonalbshah@rediffmail.com

ORCID: https://orcid.org/0000-0001-8424-0105

(c) This is an open-access article distributed under the terms of the Creative Commons Attribution Non-Commercial License (http://creativecommons.org/ licenses/by-nc/4.0/), which permits unrestricted non-commercial use, distribution, and reproduction in any medium, provided the original work is properly cited. Copyright (C) 2019 The Korean Association of Oral and Maxillofacial Surgeons. All rights reserved.
}

arch with native bone allows the closure of oronasal fistulae, a proper platform for tooth eruption, and bone support for the alar base of the nose and lip ${ }^{2}$. The incorporation of a bone graft into the alveolar cleft allows the surgeon to create a morphologically and physiologically responsive alveolus ${ }^{3}$.

The first documented bone transplant was performed by the Flemish surgeon Job van Meekeren (1611-1666), who described the successful transplant of a piece of skull bone from a dog into a defect in a human skull ${ }^{4}$. In the 1930s, the groundbreaking work of the Swiss Hermann Matti helped advance the method of transplanting autologous cancellous bone ${ }^{4}$. Ever since then, autogenous bone grafting has been used preferentially in various surgical specialties for the reconstruction of traumatic, ablative, and congenital defects ${ }^{5}$.

Many sources of bone, both autogenous and alloplastic, have been studied, compared, profiled, and abandoned, but fresh, autogenous, cancellous bone is ideal because it supplies living, immunocompatible bony cells that are essential for osteogenesis and integrate fully with the maxilla ${ }^{6}$. Various donor sites have been used to harvest cancellous bone, 
including the iliac crest, tibia, mandibular symphysis, calvarium, and rib $^{7}$. Every donor site has potential complications, so the optimal donor site remains open to debate. However, the iliac crest remains the gold standard for secondary alveolar cleft bone grafting because of its accessibility, abundance of cancellous bone, relative ease of bone harvest, ability to perform simultaneous harvest and oral procedures, and the fact that the defect is both well covered and not prone to pathological fracture ${ }^{1}$. Furthermore, placing cancellous bone from the ilium into the alveolar cleft has a predictable outcome and a high success rate ${ }^{7}$.

Classically, iliac bone grafts have been obtained using an open approach (3-4 cm incision to obtain adequate visualization, blunt dissection down to the iliac crest, and bone harvesting with an osteotome) described by Lindeman in 1915 and popularized by Wolfe and Kawamoto in $1978^{2}$. Even though the conventional open method is relatively safe and effective, concerns about an unacceptably high morbidity rate for this procedure, including impaired ambulation, significant pain, visible scar, contour deformities, sensory loss, and prolonged recovery time, have led to a search for less invasive harvesting methods ${ }^{3}$. Recently, iliac bone grafts have been obtained using minimally invasive techniques (closed approach) with different surgical devices, such as a trephine, percutaneous needle, Volkmann curette, modified bone core biopsy trocar, cylinder osteotome, manually driven osteotome, motorized CORB needle, bone grinder, grinding harvester, and mechanized coring ${ }^{2}$.

Many studies in the literature compare the conventional open technique with the various minimally invasive techniques for obtaining iliac bone grafts in terms of postoperative pain, sensory disturbance, aesthetic impairment, etc.

Although iliac crest harvesting is widely performed, donor site morbidity remains a non-trivial issue to be discussed. Therefore, the aim of this systematic review is to evaluate whether a minimally invasive technique is better than the conventional technique for harvesting iliac crest bone for secondary alveolar bone grafting in cleft palate patients in terms of morbidity at the donor site.

\section{Focused question}

Is a minimally invasive technique better than the conventional technique for harvesting iliac crest bone for secondary alveolar bone grafting in cleft palate patients in terms of morbidity at the donor site?

\section{Objective}

To evaluate and compare donor site morbidity following surgery using the minimally invasive and conventional open harvesting techniques for iliac bone for secondary alveolar bone grafting in cleft palate patients.

\section{Materials and Methods}

\section{Eligibility criteria}

1) Inclusion criteria

(1) Full-text articles describing a minimally invasive or conventional open harvesting technique for iliac bone for secondary alveolar grafting in cleft palate patients.

(2) Articles published between January 1, 2001 and June $30,2017$.

2) Exclusion criteria

(1) Articles published in languages other than English.

(2) Case reports, case series, animal studies, in vitro studies, and letters to the editor.

(3) Full-text article not available even after writing to the authors.

\section{2. $\mathrm{PICO}$}

P: patients requiring surgery for cleft palate

I: minimally invasive technique

$\mathrm{C}$ : conventional open harvesting technique

$\mathrm{O}$ : postoperative pain, duration of hospital stay, length of scar, need for postoperative medications, gait disturbance

\section{Information sources}

A comprehensive search of the literature was undertaken. A date restriction from January 1, 2001 to June 30, 2017 and language restriction to English was used in the electronic search, which included PubMed, Google Scholar, EMBASE, and an institutional library. In addition, the bibliographies of the included studies were hand searched to identify potentially eligible studies that were not captured by the electronic search. Emails were also sent to authors of potentially eligible studies to obtain additional material. A manual search of the resources in the Dr. D. Y. Patil University library was carried out. Only studies conducted in humans were considered. 


\section{Search}

The key words were used in various combinations to form search strategies.(Tables 1, 2) The search engines used PubMed, Google Scholar, EMBASE, and institutional library.

\section{Study selection}

Preliminary screening netted 274 studies, of which 223 studies were excluded in the initial screening for not meeting the eligibility criteria. Of the remaining 51 studies, 19 were removed for being duplicates. Of the remaining 32 studies, 15 were excluded based on reading the abstract. Of the 17 studies that were left, 2 were excluded because they were in a language other than English, and 2 were excluded because the authors did not mention cleft palate patients. Thus, this qualitative synthesis includes 13 studies with a total of 654 patients.

Data were extracted independently by A.S., and the data extraction was confirmed by investigators (S.S. and P.W.). At first, the studies were screened by title and abstract, and for those that fulfilled the eligibility criteria in the first screening, the second step was to obtain the full-text of the articles. Any disagreement between the two reviewers was resolved after discussion. Then a data extraction sheet was prepared.

Table 1. Keywords used for search strategies

\begin{tabular}{clc}
\hline Serial No. & \multicolumn{1}{c}{ Keywords } & Synonyms \\
\hline 1 & Iliac crest & - \\
2 & Cleft palate & - \\
3 & Bone graft & Bone transplantation \\
4 & Secondary alveolar bone grafting & - \\
5 & Minimally invasive & - \\
6 & Trephine & - \\
\hline
\end{tabular}

Aditi Saha et al: Comparison of minimally invasive versus conventional open harvesting technique for iliac bone graft in secondary alveolar bone grafting in cleft palate patients: a systematic review. J Korean Assoc Oral Maxillofac Surg 2019

\section{Data collection process}

A standard pilot form in Microsoft Excel 2010 (Microsoft, Redmond, WA, USA) was used initially. Data extraction was done for one article, and the pilot form was reviewed by an expert and finalized. This was followed by data extraction for all the articles.

\section{Data items}

The data items included were (1) study ID, (2) author name of the author(s), (3) location - place where the study was conducted, (4) year of publication - the year in which the study was published, (5) study design - retrospective/prospective/comparative, (6) setting - hospital/outpatient department, (7) sample size, (8) intervention - type of minimally invasive technique, (9) study group - type of population included in the study, (10) control - conventional technique, (11) results - result of the study, and (12) remarks - author comments (A.S., S.S., and P.W.).

\section{Results}

\section{Study selection}

This systematic review followed the PRISMA guidelines (Preferred Reporting Items for Systematic Reviews and Meta-Analyses).(Fig. 1)

\section{Study characteristics}

The studies are listed in Tables 3 and 4.

\section{Discussion}

For more than four decades, the iliac crest has been widely

Table 2. Search strategies

\begin{tabular}{|c|c|c|c|c|}
\hline Serial No. & Search strategy & $\begin{array}{l}\text { No. of } \\
\text { articles }\end{array}$ & $\begin{array}{c}\text { No. of } \\
\text { selected articles }\end{array}$ & $\begin{array}{c}\text { No. of articles after } \\
\text { duplicate removal }\end{array}$ \\
\hline 1 & Iliac crest AND cleft palate & 91 & 7 & 7 \\
\hline 2 & Iliac crest AND bone graft AND cleft palate & 85 & 8 & 1 \\
\hline 3 & Iliac crest AND secondary alveolar bone grafting & 42 & 5 & 1 \\
\hline 4 & Cleft palate AND secondary alveolar bone grafting AND minimally invasive & 2 & 0 & 0 \\
\hline 5 & Bone transplantation AND iliac crest AND minimally invasive & 32 & 4 & 0 \\
\hline 6 & Iliac crest AND bone graft harvesting AND trephine & 19 & 4 & 1 \\
\hline 7 & Others & - & - & 3 \\
\hline Total & & 274 & 28 & 13 \\
\hline
\end{tabular}

Aditi Saha et al: Comparison of minimally invasive versus conventional open harvesting technique for iliac bone graft in secondary alveolar bone grafting in cleft palate patients: a systematic review. JKorean Assoc Oral Maxillofac Surg 2019 


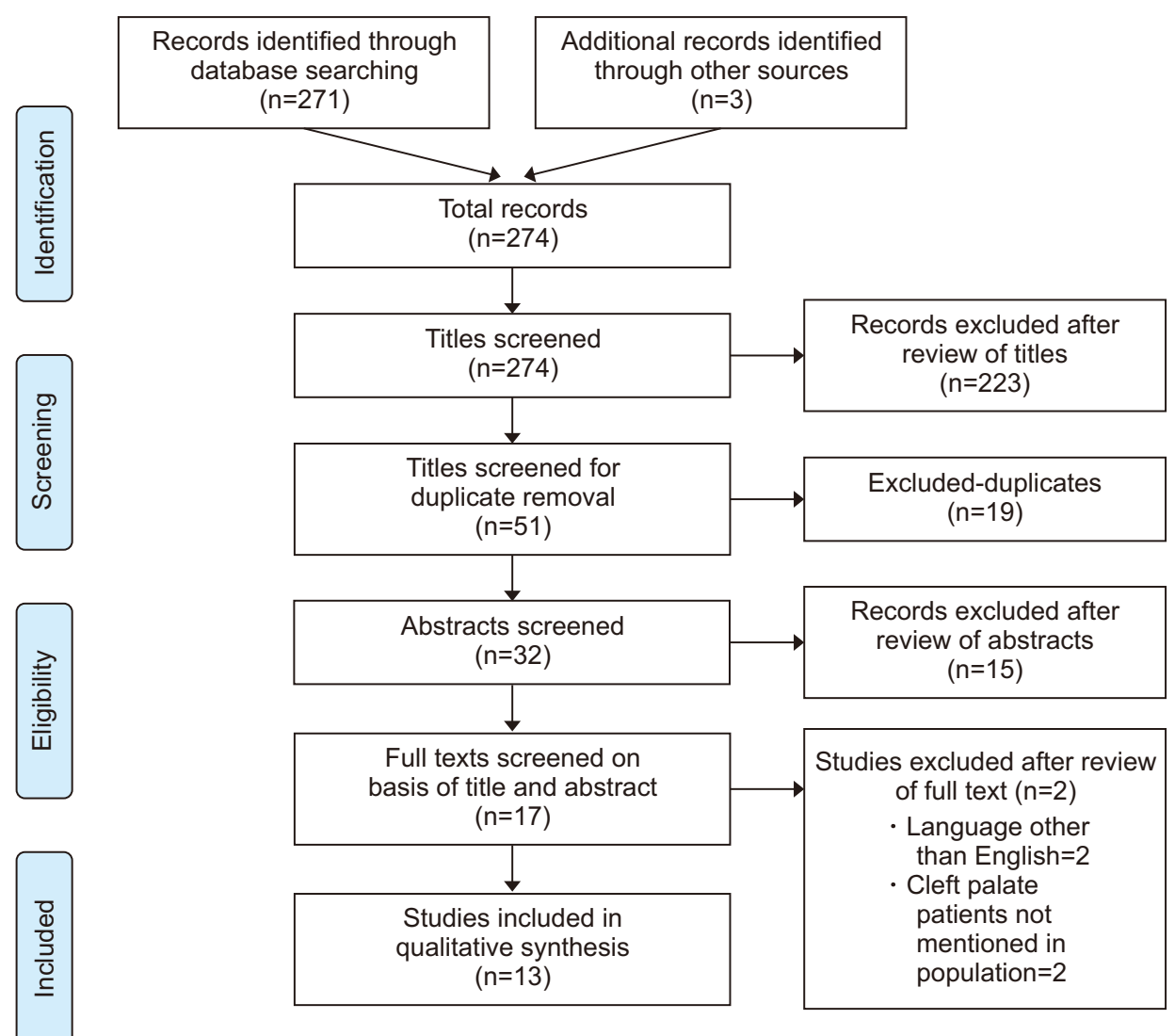

Fig. 1. PRISMA (Preferred Reporting Items for Systematic Reviews and Meta-Analyses) 2009 flow diagram.

Aditi Saha et al: Comparison of minimally invasive versus conventional open harvesting technique for iliac bone graft in secondary alveolar bone grafting in cleft palate patients: a systematic review. J Korean Assoc Oral Maxillofac Surg 2019

accepted as the gold standard bone for augmentation in orthopedic, neurosurgical, and oral and maxillofacial surgery ${ }^{8}$. Iliac crest bone grafts are by far the most commonly used autologous bone grafts. Tissue can be harvested from the anterior or posterior iliac crest. Although the iliac crest can provide an ample amount of bone-graft material and the resulting scar can be easily concealed, surgeons have long been concerned about possible complications and postoperative morbidity ${ }^{9}$, which has led to a search for minimally invasive harvesting techniques for iliac crest bone grafts ${ }^{9}$.

In 2005, Witherow et al. ${ }^{10}$ published a comparative study with 47 patients ( 23 females and 24 males) undergoing conventional open harvesting for iliac bone and 15 patients ( 8 males and 7 females) with 17 clefts undergoing a minimally invasive technique using a French's osteotome and Volkmann spoon. Follow up ranged from 18 months to 8 years. The patients were assessed by two clinicians and completed a questionnaire about symptoms related to their donor site. Their scars were measured, and any defect in the iliac crest was recorded. The researchers found that using the open technique, the average scar was $60 \mathrm{~mm}$, ranging from $30 \mathrm{~mm}$ to $125 \mathrm{~mm}, 64 \%$ of patients were asymptomatic, and 36\% complained of itching, paresthesia, hypersensitivity, or unaesthetic scars. The average length of hospital stay was 2.8 days postoperatively. Of the 15 patients who underwent the minimally invasive technique, 13 patients had unilateral (8 right and 5 left) and 2 patients had bilateral clefts. The grafts were taken from the right hip in 3 patients and left hip in 13 patients. In one patient with a large bilateral cleft both hips were opened. They had average hospital stays of 1.8 days postoperatively, and none of them required postoperative opioid analgesia. The average amount of non-opioid analgesics required was 1.8 doses, ranging from 0 to 4 doses. This study suggests that the minimally invasive technique has many advantages over the open technique, such as minimal discomfort, with the gait returning to normal within 2 to 4 days, reduced requirements for analgesics, reduced hospital stay, and virtually imperceptible scars after 1 month.

In 2006, Swan and Goodacre ${ }^{11}$ conducted a retrospective study of 73 cases (57 males and 16 females) of iliac crest bone graft harvest for secondary grafting of cleft alveoli between 1st January 1991 and 31st December 2001. The case notes were reviewed, and a postal questionnaire was sent to each patient. The questions were intended to determine postoperative convalescence, return to normal activities of daily living, and complications. A visual analogue scale (VAS) 


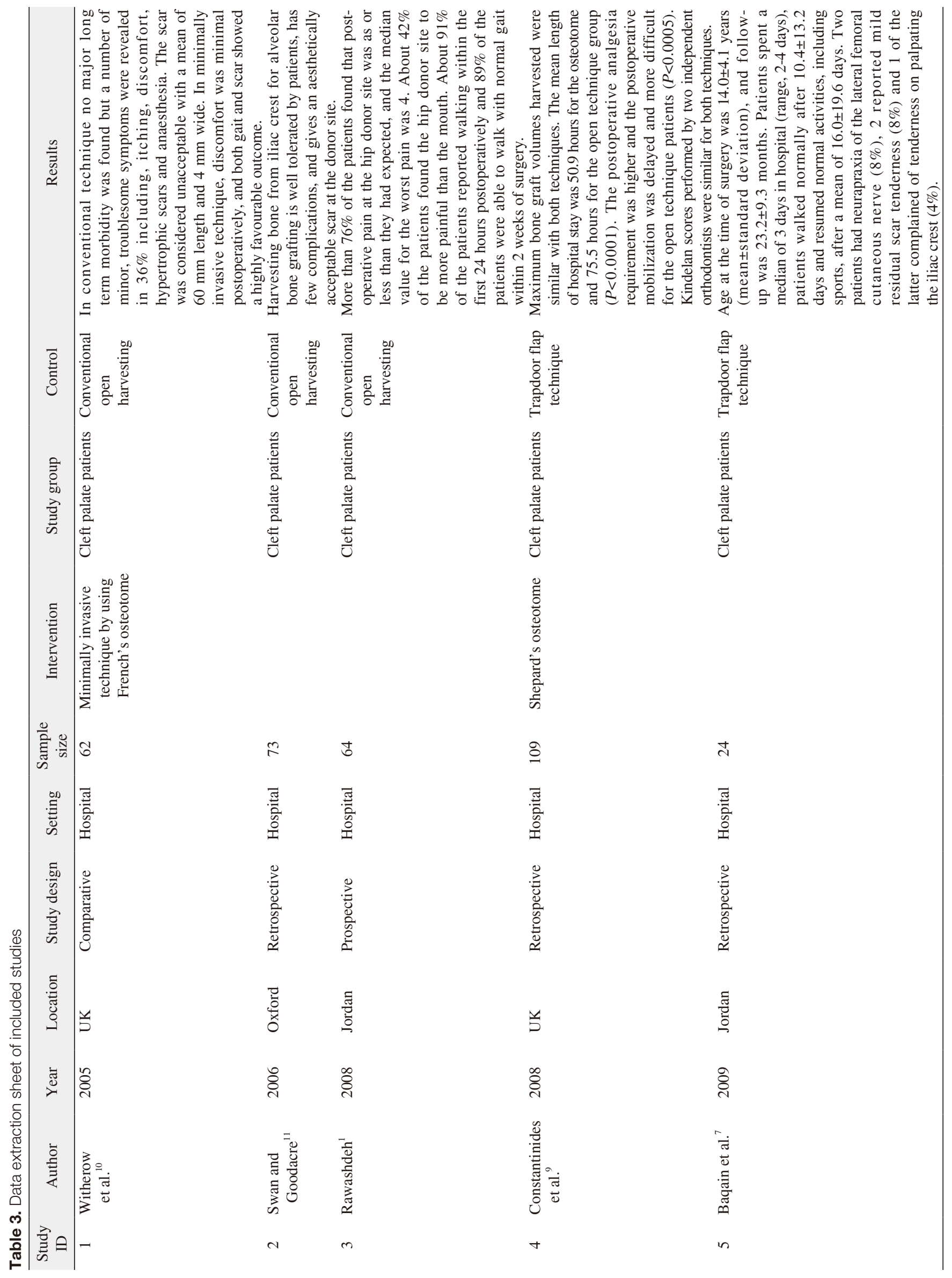




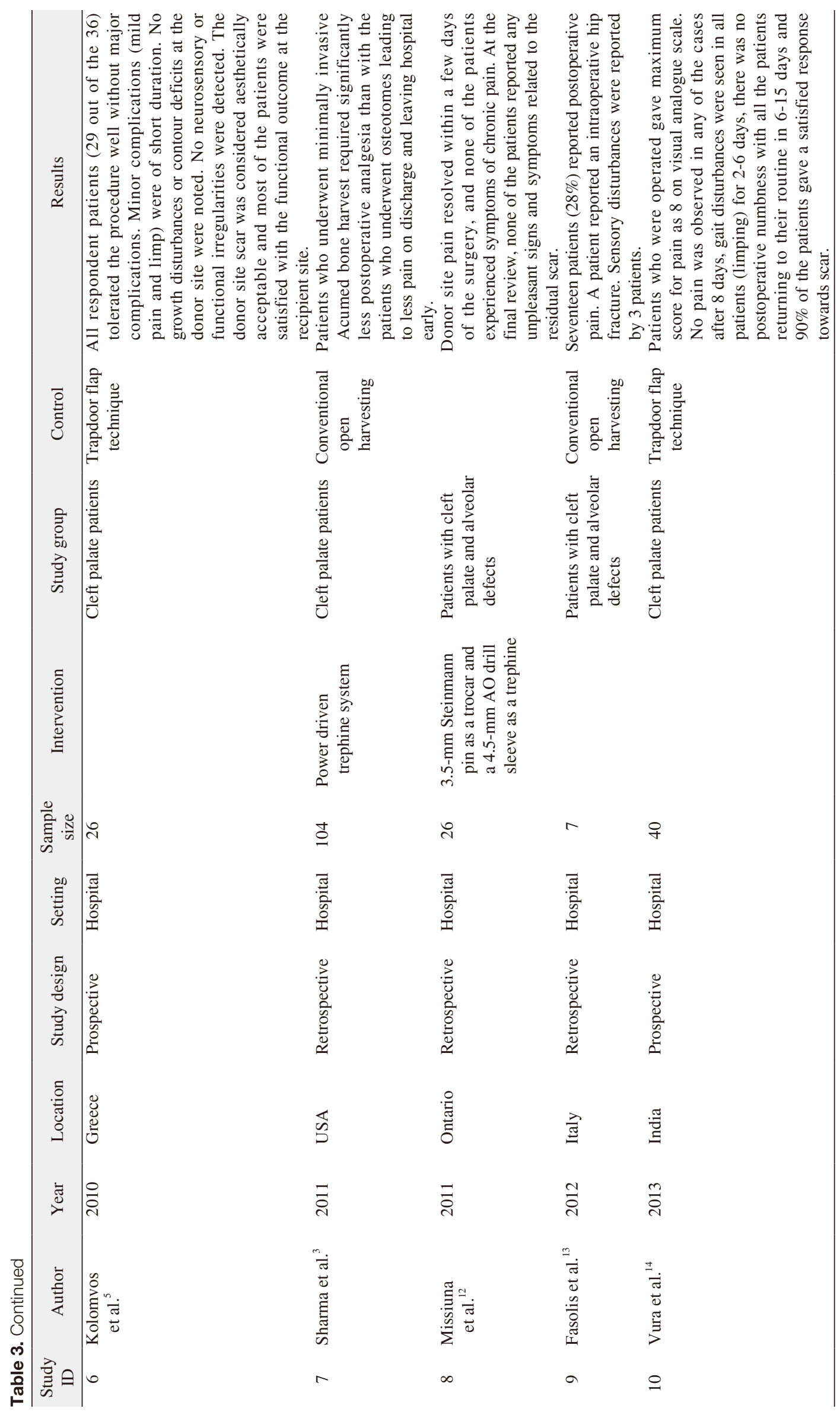




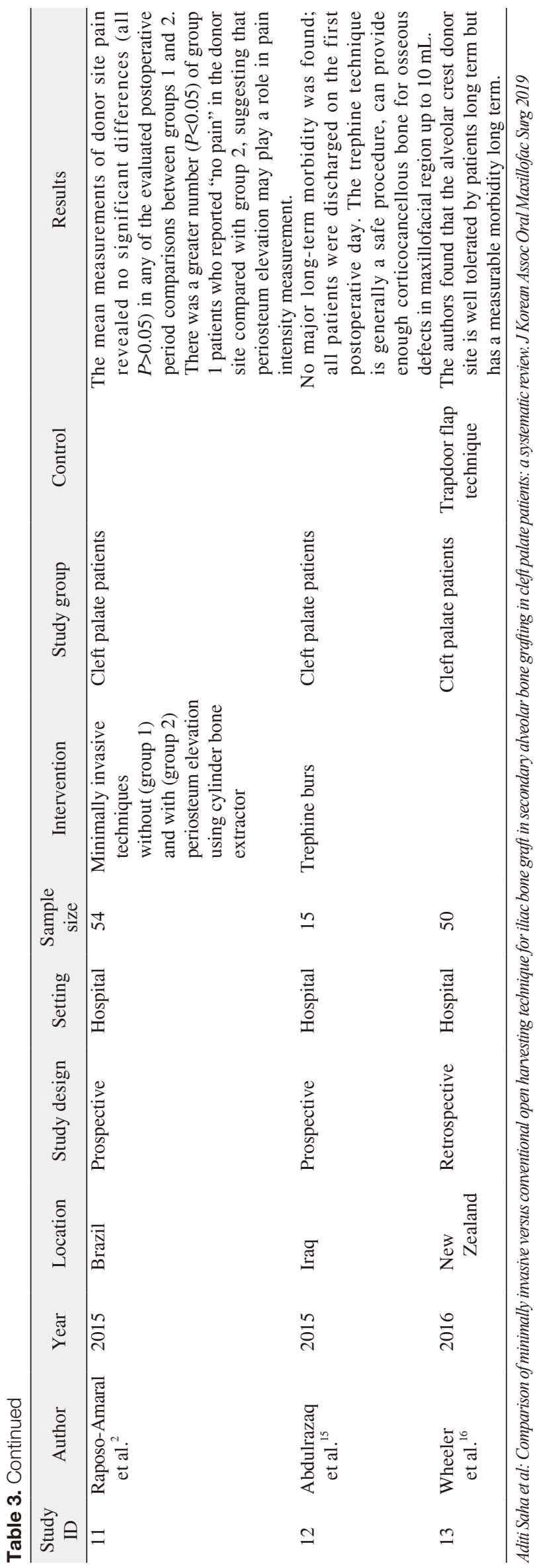

was used to establish the satisfaction of patients with the appearance of the scar at the donor site. The bone was harvested with the patient supine, thereby allowing simultaneous preparation of the recipient site. The incision was made parallel and approximately $1 \mathrm{~cm}$ inferior to the prominence of the iliac crest to ensure that the resulting scar did not lie directly over the crest. A trapdoor flap was used, and bone was harvested using a narrow osteotome. If a further graft was required, a gouge or von Volkmann's spoon was used. Before closing the trapdoor, a pediatric feeding tube was inserted within the ilium to allow the infusion of bupivacaine postoperatively. Although a vacuum drain was used in the first 40 cases, this was considered unnecessary and was subsequently abandoned without the formation of a single hematoma. Patients were encouraged to mobilize on the first postoperative day, and the bupivacaine infusions were continued for 24 to 48 hours. Of the 73 patients in the study, the questionnaire was returned by 72 (99\%). Of the clefts, 57 (78\%) were unilateral, 15 bilateral $(21 \%)$, and there was a single midline cleft (1\%). Of the unilateral clefts, 36 were left-sided (63\%) and 21 right-sided (37\%). The mean age of the patients at the time of operation was 10 years (range, 7-14). The median stay in the hospital was 3 days (range, 2-5). The median time until the child could walk normally was 7 days (range, 0-56). Thirty-seven patients $(51 \%)$ had a postoperative limp, which resolved after a median of 7 days (range, 3-56). The median length of scar was $60 \mathrm{~mm}$ (range, 40-100), and patient satisfaction was high, with a median VAS of 9/10 (range, 2-10). Five patients (7\%) reported a persistent ache at the donor site, but all such pain resolved within six months. Two patients (3\%) had superficial wound infections that were successfully treated with oral antibiotics. A single case of persistent numbness of the scar was reported, and another patient complained of a hyperaesthetic scar. A single hypertrophic scar was reported. This study concluded that harvesting bone from the iliac crest using the conventional technique for alveolar bone grafting is well tolerated by patients, has few complications, and gives an aesthetically acceptable scar at the donor site. However, no minimally invasive technique was tested in this study.

In 2008, Rawashdeh ${ }^{1}$ reported the results of a prospective study to assess donor site morbidity associated with open iliac crest bone harvesting to graft alveolar clefts in 64 patients treated between January 2000 and January 2006. Bone was harvested from the anterior iliac spine using a laterally based trapdoor flap. A curved osteotome was used to make a cut, and cancellous bone was harvested with a curette. All patients 
Table 4. Summary of findings from studies and our conclusion

\begin{tabular}{|c|c|c|}
\hline Author & Year & Result \\
\hline Witherow et al. $^{10}$ & 2005 & $\begin{array}{l}\text { Conventional technique and minimally invasive technique (French's } \\
\text { osteotome)—with minimally invasive technique, discomfort was } \\
\text { minimal postoperatively, and both gait and scar showed a highly } \\
\text { favorable outcome. }\end{array}$ \\
\hline Swan and Goodacre ${ }^{11}$ & 2006 & $\begin{array}{l}\text { Conventional technique only-few complications with an } \\
\text { aesthetically acceptable scar at the donor site. }\end{array}$ \\
\hline Rawashdeh $^{1}$ & 2008 & $\begin{array}{l}\text { Conventional open harvesting only-well tolerated by patients } \\
\text { with low morbidity. }\end{array}$ \\
\hline Constantinides et al. ${ }^{9}$ & 2008 & $\begin{array}{l}\text { Trapdoor flap technique and Shepard's osteotome-postoperative } \\
\text { analgesia requirement was higher and postoperative mobilization }\end{array}$ \\
\hline
\end{tabular}
was delayed and more difficult for the open-technique patients.

Baqain et al. ${ }^{7}$

Kolomvos et al. ${ }^{5}$ more a reasonable aesthetic outcome.

2010 Trapdoor flap technique only-the donor site scar was aesthetically acceptable, and most patients were satisfied with the functional outcome at the recipient site.

Sharma et al. ${ }^{3}$

2011 Power-driven trephine system and conventional techniquepatients who underwent the minimally invasive Acumed bone harvest required significantly less postoperative analgesia than the patients who underwent the conventional surgery.

Missiuna et al. ${ }^{12}$

2011 3.5-mm Steinmann pin as a trocar and a 4.5-mm AO drill sleeve as a trephine-donor site pain resolved within a few days of surgery, and no patients experienced chronic pain. At the final review, none of the patients reported any unpleasant signs or symptoms related to the residual scar.

Fasolis et al. $^{13}$

2012 Conventional open harvesting only-17 patients $(28 \%)$ reported postoperative pain. A patient reported an intraoperative hip fracture. Sensory disturbances were reported by 3 patients.

Vura et al. $^{14}$

Conventional open harvesting only-morbidity after harvesting bone from the iliac crest using the trapdoor technique was moderate to low with minimal complications and good tolerability and acceptance from patients.

Raposo-Amaral et al. $^{2} \quad 2015 \quad$ Minimally invasive techniques without (group 1) and with (group 2) periosteum elevation using a cylinder bone extractorno difference in pain intensity between cleft patients who had postoperative donor site pain after alveolar bone grafting. However, a greater number of patients from group 1 reported "no pain" than in group 2, suggesting that periosteum elevation and a smaller edge diameter of the bone extractor device could play a role in donor site pain.

Abdulrazaq et al. ${ }^{15}$

2015 Trephine burs-no major long-term morbidity was found; all patients were discharged on the first postoperative day. The trephine technique is generally a safe procedure that can provide enough corticocancellous bone for osseous defects in the maxillofacial region of up to $10 \mathrm{~mL}$.

Wheeler et al. ${ }^{16}$
2016 Trapdoor flap technique only-the alveolar crest donor site was well tolerated by patients long term but had measurable longterm morbidity.

\section{Remarks}

This study suggests that the minimally invasive technique has many advantages over the open technique.

No comparison group was included.

No comparison group was included.

Harvesting bone from the iliac crest using a Shepard's osteotome technique reduced the time in hospital, analgesia requirements, and postoperative donor site morbidity with no detrimental outcome.

No comparison group was included.

Bone harvesting from the anterior iliac crest was found to be a safe and reliable procedure for maxillofacial bone grafting in pediatric patients. No comparison group was included.

Minimally invasive bone-graft harvesting technique using a trephine system was better than the conventional open iliac bone harvest method, with shorter operative time, decreased requirement for pain medications, less pain on discharge, and shorter hospital stays.

Patient morbidity was significantly lower with the trephine harvest technique than with the open bone harvesting method at the anterior iliac crest, but no control group was selected for comparison.

No comparison group was included. Patients other than cleft-palate patients were included.

No comparison group was included.

No control group was included.

No control group was included.

No comparison group was included.

\footnotetext{
Aditi Saha et al: Comparison of minimally invasive versus conventional open harvesting technique for iliac bone graft in secondary alveolar bone grafting in cleft palate patients: a
} systematic review. J Korean Assoc Oral Maxillofac Surg 2019 
were reviewed by the operating surgeon at 1 week, 1 month, 3 months, 9 to 12 months, and yearly thereafter. The duration of time until postoperative ambulation and length of hospitalization were recorded. A graded VAS of 1 to 10 was used to score each patient's postoperative pain and perception of the surgical scar and procedure. Among the 64 patients, who had a mean age of 12.4 years (range, 9.2-33.1 years), 16 had bilateral clefts ( 8 males and 8 females), and 48 had unilateral clefts (26 males and 22 females). The average length of the hospital stay was 3.6 \pm 0.7 days (range, 3-5 days). More than $76 \%$ of the patients found that their postoperative pain at the hip donor site was the same or less than they had expected, and the median value for the worst postoperative pain experienced was 4 . About $42 \%$ of the patients found the hip donor site to be more painful than the mouth. About $91 \%$ of the patients reported walking within the first 24 hours postoperatively, and $89 \%$ of the patients were able to walk with their normal gait within 2 weeks of surgery. This study suggests that harvesting bone from the iliac crest is well tolerated by patients and has low morbidity when the conventional open technique is used. However, no minimally invasive technique was tested in this study.

In 2008, Constantinides et al. ${ }^{9}$ published a retrospective study about 109 patients who underwent secondary alveolar bone grafting from 1998 to 2004. Sixty-four patients (44 males and 20 females; mean age, 10.5 years; 57 unilateral, seven bilateral clefts) had their graft harvested with the open (trapdoor flap) technique, and 45 patients (28 males and 17 females; mean age, 10 years; 39 unilateral, six bilateral clefts) underwent a closed (Shepard's osteotome) technique. Patients who had their graft harvested with the open technique had their skin incision placed parallel and laterally to the iliac crest with dissection to the anterior superior iliac spine. A medially based trapdoor flap was raised, and a cancellous bone graft was harvested using an osteotome. A catheter was placed for local infusion of Marcaine $0.25 \%$ for a mean of 48.2 hours. In the closed technique, the incisions were smaller in length. Dissection to the anterior superior iliac spine was still used, and several small, circular areas of the iliac apophysis/cortex were cored out using a Shepard's osteotome. The circular cortical lid was replaced, and the wound was closed in layers. No infusion catheter was used. Hospital records for all 109 patients were reviewed and analyzed for epidemiology, alveolar cleft morphology and the presence of hard palate fistulae, hospital stay, quantity of bone graft harvested, previous medical history, and complications. Medical and nursing anesthetic notes and medication charts were also reviewed. The bone graft volume harvested varied from 1.5 to $9 \mathrm{~mL}$ for the open technique and 2 to 10 $\mathrm{mL}$ for the closed technique. Hospital stays were noticeably longer for patients who underwent the open harvest technique (range, 51-120 hours; mean, 75.7 hours) than for those who underwent the closed technique (range, 25-77 hours; mean, 50.9 hours). Twenty-nine of the 64 open-technique patients (45\%) and twenty-seven of the 45 closed-technique patients $(60 \%)$ were reported to be "mobilizing well" or "lots." Almost all patients had regular paracetamol ( $95 \%$ of open technique, $98 \%$ of closed technique patients) and a nonsteroidal anti-inflammatory analgesic ( $86 \%$ of open technique, $95 \%$ of closed technique patients). Codeine phosphate was prescribed for $64 \%$ of the closed-technique patients but only $39 \%$ of the open-technique patients. This study suggests that harvesting bone from the iliac crest using a Shepard's osteotome technique reduces time in the hospital, analgesia requirements, and postoperative donor site morbidity with no detrimental outcome.

In 2009, Baqain et al. ${ }^{7}$ conducted a retrospective study of 24 patients (14 males and 10 females) treated for alveolar clefts between 2003 and 2007. The patients and their parents were interviewed and examined by a clinician who had no access to the patients' records prior to the recall visit and had not been involved in the operation. Patients were asked about the duration of sick leave, presence and duration of postoperative limp, the use of crutches or a stick, satisfaction with their scar and the outcome of the surgical procedure, and the time taken to resume normal daily activities, including sports. In these patients, the bone was harvested between the anterior superior iliac spine and the iliac tubercle using the laterally based trapdoor flap technique with a $10 \mathrm{~mm}$ osteotome and a vacuum drain. Eight cases had bilateral alveolus clefts $(33.3 \%)$. Age at the time of operation ranged from 8 to 22 years. In all cases, cancellous bone was harvested from the left anterior iliac crest, and the amount of bone harvested was sufficient in all cases. Follow-up time ranged from 5 to 96 months. Patients spent a median of 3 days in the hospital (range, 2-4 days), walked normally after 10.4 \pm 13.2 days, and resumed normal activities, including sports, after a mean of $16.0 \pm 19.6$ days. Two patients had neurapraxia of the lateral femoral cutaneous nerve ( $8 \%), 2$ reported mild residual scar tenderness $(8 \%)$, and 1 of the latter complained of tenderness on palpating the iliac crest (4\%). This study concluded that harvesting cancellous bone from the anterior iliac crest using the conventional open technique in young patients is well tolerated, allows early resumption of normal activities, and 
has no effect on growth, minimal morbidity, and a reasonable aesthetic outcome.

In 2010, Kolomvos et al. ${ }^{5}$ reported the results of a prospective study of iliac crest bone grafting using the trapdoor technique conducted between 2001 and 2006. All patients underwent the regular preoperative assessment, including clinical and radiographic evaluation (panoramic X-ray, threedimensional computed tomography of the facial skeleton, and left anterior iliac crest X-ray). The bone was harvested from the anterior iliac crest with the trapdoor technique using a surgical drill and osteotome. Additional cancellous bone was harvested using a curette. No drains were used. Patients were re-examined 1, 6, and 12 months postoperatively, when they underwent a physical examination and were interviewed based on a questionnaire. Of the original 32 patients (20 males and 12 females), 26 (16 males and 10 females) agreed to the re-examinations and were included in the study. The mean follow-up time was 28.5 months. The median scar length was $55 \mathrm{~mm}$ (range, $40-90 \mathrm{~mm}$ ) and the median scar width was $4.8 \mathrm{~mm}$ (range, 2-15 mm). Two patients exhibited hypertrophic scars. No significant impairment of superficial sensory function and no abnormal qualities, such as hypoesthesia, hyperesthesia, anesthesia or paresthesia, were recorded in any of the patients. No gait irregularities or other mobility restrictions were recorded. Radiographs of the anterior iliac crest were taken in 16 patients, and no contour deformities or growth disturbances were revealed upon inspection. The median pain score during the first postoperative month was 1.5 (range, 0-8) at the donor site and 2 (range, 0-8) at the recipient site. The median pain score at both the donor and recipient sites 6 and 12 months after the operation was 0 . The mean time of analgesics consumption was 3 days (range, 2 days to 1 week). Gait irregularities (limp) were noted in 5 cases for a median of 9.5 days (range, 3-20 days). The median VAS score for patient satisfaction was 7.75 (range, 1-10). This study concluded that bone harvesting from the anterior iliac crest using the trapdoor technique was a safe and reliable procedure for maxillofacial bone grafting in pediatric patients.

In 2011, Sharma et al. ${ }^{3}$ conducted a retrospective study of 104 patients who underwent operative reconstruction of either bilateral or unilateral alveolar clefts between 2000 and 2009. Fifty-five patients underwent harvest with the Acumed trephine device, and 49 patients underwent conventional open iliac bone harvest with an osteotome. These two groups were compared to evaluate the operating time, hours to discharge, pain score on discharge, and administration of both narcotic and non-narcotic analgesics postoperatively in the setting of secondary alveolar cleft repair. In the conventional open technique, bone was harvested from the anterior iliac crest with a $3-\mathrm{cm}$ incision using sharp curettes or an osteotome. Drains were placed during the procedure and removed on the first or second postoperative day, before discharge. In the Acumed trephine-based technique, a 1 - to $1.5-\mathrm{cm}$ incision, just long enough to admit the trephine, was made just lateral to the crest with the skin pulled medially to hide the incision site behind the iliac crest. After dissection, the Acumed power-driven trephine was passed through this small incision a maximum of three times at different angles, which yielded sufficient bone volume. No drains were used. The 49 patients who had the conventional open osteotome technique had an average age at operation of 9.38 years (range, 6-30 years), with 24 male patients and 25 female patients. 24 of those patients had a bilateral alveolar cleft, and 25 patients had a unilateral alveolar cleft. The 55 patients who had the minimally invasive surgery had an average age at operation of 8.98 years (range, 6-19 years), with 30 male patients and 25 female patients. 20 patients presented with a bilateral alveolar cleft, and 35 patients presented with a unilateral alveolar cleft. The patients who underwent the minimally invasive Acumed bone harvest required significantly less postoperative analgesia, both narcotic and non-narcotic medications, compared with patients who underwent osteotome harvest. The Acumed patients also had significantly less pain on discharge ( 0.26 vs 3.1 on a scale of 0 to 10$)$ and left the hospital more quickly (23.3 hours vs 30.1 hours). None of the patients undergoing the Acumed harvest reported any paresthesia or shooting pain in the distribution of the lateral femoral cutaneous nerve. This study concluded that the minimally invasive bone graft harvest technique using the trephine system is a good alternative to the conventional open iliac bone harvest method for patients undergoing secondary alveolar cleft repair, with shorter operative time, decreased requirement for pain medications, less pain on discharge, and shorter hospital stays.

In 2011, Missiuna et al. ${ }^{12}$ conducted a retrospective study of 26 patients (18 males and 8 females) who underwent a minimally invasive transcrestal mid-iliac bone graft procurement technique between May 2003 and December 2007 for alveolar defects and cleft palate. The technique was performed by making a $1-\mathrm{cm}$ long skin incision $10 \mathrm{~mm}$ distal to the iliac margin that was then mobilized over the crest. Bone was harvested using a 3.5-mm Steinmann pin as a trocar and a 4.5-mm AO drill sleeve as a trephine. Drains were not used. A questionnaire to assess postoperative pain, dysesthesia, 
paresthesia, the status of the donor-site wound, and patient satisfaction was given to the patients in the clinic or sent by mail. The postoperative follow-up period was 3 to 57 months. The duration of pain experienced at the donor site was 0 to 56 days. The $69.2 \%$ were completely without pain in 7 days. The mean intensity of pain severity on a scale of 0 to 10 was 4 . The length of the scar was $11.9 \mathrm{~mm}$; the mean width was $1.7 \mathrm{~mm}$. Gait disturbances in the form of discomfort and slight limp on ambulation were reported by 11 patients (42.3\%) for a mean duration of 14 days. No hyperpigmented, hypertrophic, or keloid scars were observed or reported. The researchers concluded that the minimally invasive trephine method can be used when a small cancellous bone graft is needed because the patient morbidity was significantly lower with the trephine harvest technique than with the open bone harvesting method.

In 2012, Fasolis et al. ${ }^{13}$ conducted a retrospective study of 7 patients with cleft lip and palate who had undergone secondary alveolar bone grafting. An incision was placed $1 \mathrm{~cm}$ behind the anterior superior iliac spine and $1 \mathrm{~cm}$ lateral to the height of the crest. Bone was harvested from the anterior iliac crest using the open technique of placing a surgical drill and osteotome. A questionnaire about the level and duration of postoperative pain, residual pain, sensory disturbance, functional limitations, and cosmetic appearance was given to patients. The follow-up period varied between 32 and 84 months, with an average follow-up of 48.78 months. The mean age of patients was 13.28 years. The average length of the hospital stay was 4.9 days. The $72 \%$ of patients reported no postoperative pain at the hip donor site, whereas $28 \%$ suffered pain. The average graded VAS score for pain was 5.5. The average duration of problems walking, such as gluteal gait, was 4.24 days. The average mature scar length was $44.67 \mathrm{~mm}$. The average graded VAS score for satisfaction with the procedure was 7.38. This study suggests that harvesting bone from the iliac crest using the conventional open technique is well tolerated by patients and has low morbidity. However, no minimally invasive technique was tested in this study.

In 2013, Vura et al. ${ }^{14}$ published a prospective study of 40 patients who were treated with secondary alveolar bone grafting using the anterior iliac crest as the donor site from July 2008 to March 2013. A 3- to 5-cm incision was placed $2 \mathrm{~cm}$ away from the anterior superior iliac spine. Bone was harvested using the medial trapdoor technique with the help of a small osteotome and a gouge. Patients were given a questionnaire and reviewed postoperatively after 1 week, 15 days, 1 month, 3 months, and 9 to 12 months. The age at the time of surgery ranged from 8 to 30 years. The average duration of pain was $6.32 \pm 0.9$ days. A VAS was used to assess the severity of pain, and the mean grading was $7.68 \pm 0.47$, with pain in the hip region exceeding that in the oral region. The average duration of limping was $3.52 \pm 1.38$ days, and the period required to walk normally was $4.6 \pm 1.41$ days. The number of days taken to return to normal activity was $8.36 \pm 1.93$ days. The $92 \%$ of patients were satisfied with their postoperative scar. No case of infection or paresthesia was reported. This study concluded that morbidity after harvesting bone from the iliac crest using the trapdoor technique was moderate to low, with minimal complications, and that the technique was well tolerated with good acceptance from patients. However, no minimally invasive technique was tested in this study.

In 2015, Raposo-Amaral et al. ${ }^{2}$ reported the results of a prospective study of 54 patients (26 males and 28 females) with cleft lip and palate who underwent iliac crest bone grafting for alveolar cleft repair between 2011 and 2013. The patients were randomly divided into 2 groups: bone graft harvested by minimally invasive techniques without periosteum elevation (group $1 ; n=27$ ) and with periosteum elevation (group 2; n=27). Bone grafts were harvested from the anterior superior iliac crest using 2 different cylinder bone extractors and different techniques. In group 1, a 1.5- to 2-cm incision was made, and bone was harvested using a 5-mm cylinder bone extractor device. In group 2, a 1.5- to 2-cm incision was made, and bone was harvested using an 8 -mm cylinder bone extractor device. The mean measurements of donor site pain revealed no significant differences between groups 1 and 2 in any of the evaluated postoperative period comparisons. A greater number of group 1 patients than group 2 patients reported "no pain" at the donor site, suggesting that periosteum elevation could play a role in pain intensity measurement.

In 2015, Abdulrazaq et al. ${ }^{15}$ reported the results from a prospective study of 15 patients treated from June 2013 to December 2014. The ages varied from 9 to 43 years (mean, 18.6 years). A trephine with burs of different sizes and lengths was used. The incision was made over the iliac tubercle. The length of the incision depended on the number of holes: 1.5 $\mathrm{cm}$ for 1 hole, $2.5 \mathrm{~cm}$ for 2 holes, $3 \mathrm{~cm}$ for 3 holes, and $4 \mathrm{~cm}$ for 4 holes. All holes through the bone were made by a single bur in the iliac crest between the inner and outer tables to harvest a cylinder of cancellous bone. The surgical site was closed in layers without a drain. Postoperative complications included seroma in 1 patient $(5.6 \%)$, wound dehiscence in 1 patient $(5.6 \%)$, and a hypertrophic scar in 1 patient $(5.6 \%)$. 
No patients reported pain or problems walking during the first postoperative week. This study concluded that despite its small sample size, the trephine technique could be deemed a short, simple procedure with no major complications that can be used to obtain material for small alveolar defects; it allows early mobilization and discharge of the patients from the hospital. However, this study included no control group.

In 2016, Wheeler et al. ${ }^{16}$ conducted a retrospective study to accurately assess donor site morbidity from iliac crest bone harvesting for secondary bone grafting in patients with cleft lip and palate alveolar defects. Fifty patients (32 males and 28 females) were included. The trapdoor approach was used, and bone was harvested using an osteotome. A questionnaire with a scale was used to determine patients' level of satisfaction with the donor site. The follow-up period averaged 4 years. The average scar length was $5.4 \mathrm{~cm}$, and a third of patients had some minor palpable bony irregularities of the iliac crest. This study concluded that the alveolar crest donor site is well tolerated by patients but has measurable morbidity in the long term. This study included no control group.

All the studies reported here were conducted between 2005 and 2016. The 13 studies included in this review comprise 5 prospective studies, 7 retrospective studies, and 1 comparative study and have a global distribution. These studies report results from a total of 654 cleft palate patients who underwent surgery in a hospital setup using the conventional open (trapdoor) technique or a minimally invasive technique (French's osteotome, Shepard's osteotome, Acumed trephine, 3.5-mm Steinmann pin as a trocar and a $4.5-\mathrm{mm}$ AO drill sleeve as a trephine, cylinder bone extractor, or trephine burs). Minimally invasive techniques were used in 6 studies, of which 3 did not include a comparison group. The length of hospital stay postoperatively was longer with the conventional open technique (2-4 days) than with any of the minimally invasive techniques (1-3 days). The mean VAS score for donor-site pain was higher with the conventional open technique than with any of the minimally invasive techniques. Likewise, the average scar length was longer with the conventional technique $(50 \mathrm{~mm})$ than with the minimally invasive techniques $(11.9 \mathrm{~mm})$, as was the period required to walk normally without gait disturbances. The requirement for analgesics was less with the minimally invasive techniques than with the open conventional technique. Most patients were satisfied with their postoperative scar, irrespective of the technique used. Very few ( $2 \%$ ) complications, such as superficial donor site infections, persistent numbness of scar, neuropraxia of the lateral femoral cutaneous nerve, seroma, wound dehiscence, and hypertrophic scars, were reported with any technique.

In this review, 6 studies (less than 50\%) used a minimally invasive technique, and 3 of those did not compare it with the conventional open harvesting technique. Seven studies reported results from the conventional open harvesting technique without comparing it with a minimally invasive technique. The parameters for assessing donor site morbidity differed in all studies. One study had a sample size of only 7 subjects. The number of studies comparing two techniques is too small to provide meaningful data.

\section{Conclusion}

After a thorough comparison of the results, the minimally invasive bone harvest techniques appear to be better than the conventional open iliac bone harvest method for patients undergoing secondary alveolar cleft repair because they offer shorter operative times, decreased requirements for pain medication, less pain on discharge, and a shorter hospital stay.

One of the drawbacks of this systematic review is the exclusion of articles comparing only minimally invasive techniques and conventional open harvesting. Future researchers could undertake a long-term, multi-center, prospective, randomized controlled trial to compare the various techniques and determine which is better.

\section{ORCID}

Aditi Saha, https://orcid.org/0000-0002-2660-713X

Sonal Shah, https://orcid.org/0000-0001-8424-0105

Pushkar Waknis, https://orcid.org/0000-0002-8230-6075

Prathamesh Bhujbal, https://orcid.org/0000-0001-7772-8567

Sharvika Aher, https://orcid.org/0000-0002-7613-7631

Vibha Vaswani, https://orcid.org/0000-0002-7504-7271

\section{Authors' Contributions}

A.S. participated in data collection and wrote the manuscript. S.S. and P.W. participated in the study design and performed the statistical analysis. P.B., S.A., and V.V. participated in the study design and coordination and helped to draft the manuscript. All authors read and approved the final manuscript. 


\section{Conflict of Interest}

No potential conflict of interest relevant to this article was reported.

\section{References}

1. Rawashdeh MA. Morbidity of iliac crest donor site following open bone harvesting in cleft lip and palate patients. Int J Oral Maxillofac Surg 2008;37:223-7.

2. Raposo-Amaral CA, Denadai R, Chammas DZ, Marques FF, Pinho AS, Roberto WM, et al. Cleft patient-reported postoperative donor site pain following alveolar autologous iliac crest bone grafting: comparing two minimally invasive harvesting techniques. J Craniofac Surg 2015;26:2099-103.

3. Sharma S, Schneider LF, Barr J, Aarabi S, Chibbaro P, Grayson B, et al. Comparison of minimally invasive versus conventional open harvesting techniques for iliac bone graft in secondary alveolar cleft patients. Plast Reconstr Surg 2011;128:485-91.

4. Schaaf H, Lendeckel S, Howaldt HP, Streckbein P. Donor site morbidity after bone harvesting from the anterior iliac crest. Oral Surg Oral Med Oral Pathol Oral Radiol Endod 2010;109:52-8.

5. Kolomvos N, Iatrou I, Theologie-Lygidakis N, Tzerbos F, Schoinohoriti $\mathrm{O}$. Iliac crest morbidity following maxillofacial bone grafting in children: a clinical and radiographic prospective study. J Craniomaxillofac Surg 2010;38:293-302.

6. Rawashdeh MA, Telfah $\mathrm{H}$. Secondary alveolar bone grafting: the dilemma of donor site selection and morbidity. Br J Oral Maxillofac Surg 2008;46:665-70.

7. Baqain ZH, Anabtawi M, Karaky AA, Malkawi Z. Morbidity from anterior iliac crest bone harvesting for secondary alveolar bone grafting: an outcome assessment study. J Oral Maxillofac Surg 2009;67:570-5

8. Kessler P, Thorwarth M, Bloch-Birkholz A, Nkenke E, Neukam FW. Harvesting of bone from the iliac crest--comparison of the anterior and posterior sites. Br J Oral Maxillofac Surg 2005;43:51-6.

9. Constantinides J, Chhabra P, Turner PJ, Richard B. A comparison of Shepard's osteotome versus trapdoor flap technique to harvest iliac crest bone for secondary alveolar bone grafting. Cleft Palate Craniofac J 2008;45:347-52.

10. Witherow H, Lee RKL, Blenkinsopp PT, Waterhouse N. Comparison of a modified minimally invasive with an open technique following harvesting of cancellous iliac bone. Eur J Plast Surg 2005;28:268-71.

11. Swan MC, Goodacre TE. Morbidity at the iliac crest donor site following bone grafting of the cleft alveolus. Br J Oral Maxillofac Surg 2006;44:129-33.

12. Missiuna PC, Gandhi HS, Farrokhyar F, Harnett BE, Dore EM, Roberts B. Anatomically safe and minimally invasive transcrestal technique for procurement of autogenous cancellous bone graft from the mid-iliac crest. Can J Surg 2011;54:327-32.

13. Fasolis M, Boffano P, Ramieri G. Morbidity associated with anterior iliac crest bone graft. Oral Surg Oral Med Oral Pathol Oral Radiol 2012;114:586-91.

14. Vura N, Reddy K R, R S, G R, Kaluvala VR. Donor site evaluation: anterior iliac crest following secondary alveolar bone grafting. J Clin Diagn Res 2013;7:2627-30.

15. Abdulrazaq SS, Issa SA, Abdulrazzak NJ. Evaluation of the trephine method in harvesting bone graft from the anterior iliac crest for oral and maxillofacial reconstructive surgery. J Craniofac Surg 2015;26:e744-6.

16. Wheeler J, Sanders M, Loo S, Moaveni Z, Bartlett G, Keall H, et al. Iliac crest donor site for children with cleft lip and palate undergoing alveolar bone grafting: a long-term assessment. J Craniofac Surg 2016;27:598-601.

How to cite this article: Saha A, Shah S, Waknis P, Bhujbal P, Aher S, Vaswani V. Comparison of minimally invasive versus conventional open harvesting technique for iliac bone graft in secondary alveolar bone grafting in cleft palate patients: a systematic review. J Korean Assoc Oral Maxillofac Surg 2019;45:241-253. https://doi.org/10.5125/jkaoms.2019.45.5.241 